\title{
Two-way coupling model for shock-induced laminar boundary-layer flows of a dusty gas
}

\begin{abstract}
The present paper describes a numerical two-way coupling model for shock-induced laminar boundary-layer flows of a dust-laden gas and studies the transverse migration of fine particles under the action of Saffman lift force. The governing equations are formulated in the dilute twophase continuum framework with consideration of the finiteness of the particle Reynolds and Knudsen numbers. The full Lagrangian method is explored for calculating the dispersedphase flow fields (including the number density of particles) in the regions of intersecting particle trajectories. The computation results show a significant reaction of the particles on the two-phase boundary-layer structure when the mass loading ratio of particles takes finite values.
\end{abstract}

Keywords Shock wave · Dusty gas · Two-phase boundary layer · Two-way coupling $\cdot$ Numerical modeling

\section{Introduction}

Gas-particle flows are commonly encountered in both natural and industrial processes. Sand-dust storms, tornados, volcano eruptions, fluidized beds, coal classifiers, powder conveyers and particle-laden jets are only few examples of the applications involving two-phase flows. One important engineering application is the prediction and prevention of dust fires and explosions in plants, storerooms and coal mine. It is well known that many organic or metallic powders (such

The project supported by the National Natural Science Foundation of China (90205024) and Russian Foundation for Basic Research (RFBR and (RFBR-NSFC-39004)

The English text was polished by Yunming Chen

B.Y. Wang $(\varangle) \cdot$ Y. Xiong

LNM, Institute of Mechanics, Chinese Academy of Sciences,

Beijing 100080, China

E-mail: wby@imech.ac.cn

A.N. Osiptsov

Institute of Mechanics, Moscow State University,

119899 Moscow, Russia as cornstarch, coal, aluminum and magnesium) suspended in air form explosive mixtures due to huge specific surface area of fine dispersed particles. Dust fires or explosions may occur if the ignition-source initiation energy is high enough. Usually, explosive particles are deposited down or piled up on the floor and it is quite difficult to ignite them. However, the particles may be aerodynamically entrained into the air flow when a shock wave, which can be induced by a primary gaseous explosion, propagates over the deposit layer. This results in the formation of a dust cloud and the increase of the air temperature behind the shock front. In other words, due to this double factor, the moving shock wave is a mechanism for enhancing the possibility of dust explosions. In particular, in tubes of large diameter and length, deflagration in a dust-air mixture may transit to a self-sustained detonation [1], which is a matter of concern both in industrial facilities used for processing powder materials and in coal mines. Compared to gaseous mixtures, dust-air suspensions have extremely high explosion limits [2]. In estimating explosion hazards, various critical parameters (maximum pressure, rate of pressure rise, time delay, detonation velocity, etc.) depend on dust size and concentration. The effect of dust rise behind shock waves moving over a deposit bed of particles is well known from many experimental observations (see, for example Refs. [3,4]).

In this study, an attempt is undertaken to model the flow fields of the carrier and dispersed phase in the laminar region of a near-wall two-phase flow induced by a normal shock wave traveling at a constant speed over an eroded surface of a layer of deposited particles with consideration of the Saffman lift force exerted on the particles and the particle reaction on the carrier phase. The simplest possible boundary condition, namely, a constant mass flux of the particles $N^{*}$ is specified on the surface of the eroded particle bed.

The transverse migration of single fine particles from the deposit surface under the action of lift forces (shear-induced Saffman force and Magnus force) was previously considered [5-8]. In those papers, only particle trajectories have been investigated but the distribution of particle concentration has not been obtained. The calculation of particle concentration 
in the near-wall region is a fairly difficult problem due to the onset of crossing particle trajectories and the resulting nonuniqueness of particle parameters. Recently, Thevand and Daniel [9] calculated the characteristics of two-phase shocktube boundary-layer flows by using an Eulerian approach but their method is applicable only in the flow region where the particle trajectories do not intersect each other. With a novel full Lagrangian method [10], Wang et al. [11-13] performed numerical simulations of shock-induced two-phase boundary-layer flows within the one-way coupling model. In the present work, this approach is developed to take into account the two-way coupling between the carrier and dispersed phases and to study in detail the effect of particles on the two-phase flow patterns.

\section{Mathematical formulation}

For the two-dimensional boundary layer of a dusty gas, induced by a normal shock wave traveling along a plane wall deposited with loose fine particles, the flow is laminar down to the point of transition to turbulence. It is assumed that ahead of the shock front, over a thin bed of deposit, there is clean air whose temperature is the same as the constant temperature of the wall and the particle bed $T_{w}^{*}$. When the shock wave velocity $U_{\infty}^{*}$ (or shock Mach number $M a$ ) is constant, the two-phase flow can be regarded as a steady one in the coordinates fitted to the shock front.

\subsection{Basic Assumptions}

For the gas-particle suspension, usual assumptions of the dilute dusty-gas model [14] are adopted. The carrier phase is a perfect viscous compressible gas. The dispersed phase consists of identical solid spheres with radius $\sigma^{*}$ and mass $m^{*}$. There is no temperature gradient inside the particles. The Brownian motion, mutual collisions and particle volume concentration are negligible (accordingly, the dispersed phase can be treated as a zero-pressure fluid). There is no mass transfer between the gas and particles, since neither chemical reactions nor phase transitions occur in the suspension system.

Due to different inertial and thermal properties of the two phases, the interphase momentum and energy exchange should be taken into account. The drag and Saffman force as well as the heat flux exerted on a single spherical particle can be expressed in the following forms [15-18]

$f^{*}=f_{D}^{*}+f_{S a f}^{*}$,

$q^{*}=4 \pi \sigma^{*} k^{*}\left(T^{*}-T_{\mathrm{s}}^{*}\right) G$.

Here the aerodynamic drag $f_{D}^{*}$ and shear-induced lift force $f_{\text {Saf }}^{*}$ are, respectively, given as

$\boldsymbol{f}_{D}^{*}=6 \pi \sigma^{*} \mu^{*}\left(\boldsymbol{V}^{*}-\boldsymbol{V}_{\mathrm{s}}^{*}\right) D$,

$\boldsymbol{f}_{\text {Saf }}^{*}=-6.46 \sigma^{* 2}\left(\rho^{*} \mu^{*}\left|\frac{\partial u^{*}}{\partial y^{*}}\right|\right)^{1 / 2} H\left(u^{*}-u_{\mathrm{s}}^{*}\right) \boldsymbol{j}$.
Due to the finiteness of the particle Reynolds and Knudsen numbers in the local flow around the particle, the above expressions contain three correction functions (hereafter the subscript "s" denotes solid particles):

(a) $D=\left(1+R e_{\mathrm{s}}^{2 / 3} / 6\right) \Phi\left(M a_{\mathrm{s}}, R e_{\mathrm{s}}\right)$,

where

$$
\begin{aligned}
& \Phi=\left(1+\exp \left(-0.427 M a_{\mathrm{s}}^{-4.63}-3 R e_{\mathrm{s}}^{-0.88}\right)\right) / \psi \\
& \psi=1+K n_{\mathrm{s}}\left(2.57+0.68 \exp \left(-1.86 / K n_{\mathrm{s}}\right)\right) ;
\end{aligned}
$$

(b) $\quad G=\left(1+0.3 \operatorname{Pr}^{1 / 3} \operatorname{Re}_{\mathrm{s}}^{1 / 2}\right) / \Psi$,

where

$$
\Psi=1+2.30 K n_{\mathrm{s}}\left(1+0.3 \operatorname{Pr}^{1 / 3} \operatorname{Re}_{\mathrm{s}}^{1 / 2}\right) / \operatorname{Pr} ;
$$

(c) $H=0.4687\left[1-\exp \left(-0.1 R e_{\mathrm{s}}\right)\right]\left(R e_{\mathrm{g}} / R e_{\mathrm{s}}\right)^{1 / 2}$

$$
+\exp \left(-0.1 R e_{\mathrm{s}}\right)
$$

or $H=0.0741 R e_{\mathrm{g}}^{1 / 2}$ at $R e_{\mathrm{s}}>40$.

Here, as usual, $\boldsymbol{V}^{*}, T^{*}, \rho^{*}, \mu^{*}, k^{*}$ are the velocity vector (its $x^{*}$ - and $y^{*}$ - components are $u^{*}$ and $v^{*}$ ), temperature, density and viscosity, heat conductivity. Clearly, in addition to the gas Prandtl number $\mathrm{Pr}$, the interphase momentum and energy exchange is controlled by four dimensionless parameters (the slip Reynolds number $R e_{\mathrm{s}}$, the shear Reynolds number $R e_{\mathrm{g}}$, the slip Mach number $M a_{\mathrm{s}}$ and the Knudsen number $K n_{\mathrm{s}}$ ). In the near-wall two-phase flow, the gas and particles are far from equilibrium. The velocity slip and the temperature jump between the gas and the particles lead to significant interphase momentum and heat exchanges. For the gas-particle flow under consideration, due to relatively slow change of the slip velocity, additional forces (for example, pressure gradient force, carried mass force, and Basset force) do not come into play [19]. Besides, due to zero rotation velocity of the particles, Magnus force is not included though it can be one of the mechanisms accounting for particle migration.

\subsection{Governing equations}

As mentioned in Ref. [11], in the shock-induced two-phase boundary layer, multiple intersections of particle trajectories occur, which results in the onset of the non-uniqueness of the particle flow parameters. Standard Eulerian approaches for both phases, sometimes still used in the literature (see, for example Ref. [9]), a priori forbid any intersections of particle trajectories and this may result in the insufficient accuracy of calculations and distort the flow patterns. To take correctly into account the possibility of particle trajectory intersections and the reaction of the particles on the carrier phase, we developed a new hybrid approach: the parameters of the carrier phase are calculated on the Eulerian grid while the 
parameters of the dispersed phase (including the concentration) are calculated along the particle trajectories using full Lagrangian method [10]. To calculate the particle reaction, we use global iterations with respect to the terms describing the interphase momentum and energy exchange.

To obtain the governing equations, the stretched boundary-layer coordinate $\eta$ and dimensionless flow variables are introduced (the superscript $*$ refers to the dimensional quantities)

$$
\begin{array}{ll}
x=\frac{x^{*}}{l^{*}}, & \eta=\frac{y^{*}}{l^{*}} \sqrt{R e}, \\
t=\frac{t^{*} u_{+}^{*}}{l^{*}}, & u=\frac{u^{*}}{u_{+}^{*}}, \\
v=\frac{v^{*}}{u_{+}^{*}} \sqrt{R e}, & T=\frac{T^{*}\left(U_{\infty}^{*}-u_{+}^{*}\right)+u_{+}^{*} T_{w}^{*}-U_{\infty}^{*} T_{+}^{*},}{\left(T_{w}^{*}-T_{+}^{*}\right) u_{+}^{*}} \\
\rho=\frac{\rho^{*}}{\rho_{+}^{*}}, & p=\frac{p^{*}}{\rho_{+}^{*} u_{+}^{* 2}}, \\
n_{\mathrm{s}}=\frac{n_{\mathrm{s}}^{*} u_{+}^{*}}{N^{*} \sqrt{R e}}, &
\end{array}
$$

where $p$ and $n_{\mathrm{s}}$ are the gas pressure and particle number density; $N^{*}$ is the normal (upward) number flux of the particles on the wall; $l^{*}=m^{*} u_{+}^{*} / 6 \pi \sigma^{*} \mu_{+}^{*}$ is the phase velocity relaxation length; $R e=\rho_{+}^{*} u_{+}^{*} l^{*} / \mu_{+}^{*}$ is the flow Reynolds number based on the relaxation length. Besides, in this paper, it is assumed that, in the boundary layer, the transport coefficients of the gas are linear functions of the gas temperature $\left(\mu^{*} / \mu_{+}^{*}=k^{*} / k_{+}^{*}=K T^{*} / T_{+}^{*}\right)$. In addition, it is assumed that the particles leave the eroded surface of the particle bed with zero normal velocity but the number flux of the particles takes a constant finite value $N^{*}=$ const. The gas flow parameters in the inviscid region immediately behind the shock front (denoted with the subscript + ) are related with the known gas parameters ahead the front by the standard Rankine-Hugoniot conditions.

In the shock-fitted coordinates $(x, \eta)$, the governing equations of the carrier phase can be written in the following form:

$$
\begin{aligned}
\frac{\partial \rho u}{\partial x}+\frac{\partial \rho v}{\partial \eta}=0, & \\
\rho\left(u \frac{\partial u}{\partial x}+v \frac{\partial u}{\partial \eta}\right)= & K \frac{\partial}{\partial \eta}\left(\theta \frac{\partial u}{\partial \eta}\right) \\
& -\alpha K \theta \sum_{m} n_{\mathrm{s} m}\left(u-u_{\mathrm{s} m}\right) D_{m},
\end{aligned}
$$

$$
\begin{aligned}
\rho\left(u \frac{\partial T}{\partial x}+v \frac{\partial T}{\partial \eta}\right) \\
=\frac{K}{\operatorname{Pr}} \frac{\partial}{\partial \eta}\left(\theta \frac{\partial T}{\partial \eta}\right)+(a-1) E c \\
\quad \times\left[K \theta\left(\frac{\partial u}{\partial \eta}\right)^{2}+\alpha K \theta \sum_{m} n_{\mathrm{s} m}\left(u-u_{\mathrm{s} m}\right)^{2} D_{m}\right] \\
\quad-\frac{2}{3 \operatorname{Pr}} \alpha K \theta \sum_{m} n_{\mathrm{s} m}\left(T-T_{\mathrm{s} m}\right) G_{m}, \\
\frac{\partial p}{\partial \eta}=0, \\
\rho \theta=1,
\end{aligned}
$$

where, for convenience, a new variable is defined as $\theta=$ $T^{*} / T_{+}^{*}=[(d-1) T+a-d] /(a-1)$. In Eqs.(1), five dimensionless parameters are introduced: $a=U_{\infty}^{*} / u_{+}^{*}, d=$ $T_{w}^{*} / T_{+}^{*}, \operatorname{Pr}=c_{p}^{*} \mu_{+}^{*} / k_{+}^{*}, E c=u_{+}^{* 2} / c_{p}^{*}\left(T_{w}^{*}-T_{+}^{*}\right)$, and $\alpha=$ $m^{*} N^{*} \sqrt{\operatorname{Re}} / \rho_{+}^{*} u_{+}^{*}$. The subscript $m$ here denotes the number of particle trajectories crossing at the point of space under consideration. The boundary conditions are given as

$\eta=0: \quad u=a, \quad v=0, \quad T=a$,

$\eta=\infty: \quad u=1, \quad T=1$,

$x=0, \quad \eta>0: \quad u=1, \quad v=0, \quad T=1$.

To resolve the problems associated with the intersections of particle trajectories, we introduce the Lagrangian variables $\left(t, x_{0}\right)$, where $x_{0}$ is the dimensionless coordinate of the origin of a particle trajectory on the wall and $t$ the dimensionless time of particle motion along this trajectory. In the Lagrangian variables, the governing equations of the dispersed phase take the form:

$$
\begin{aligned}
& \frac{\mathrm{d} x_{\mathrm{s}}}{\mathrm{d} t}=u_{\mathrm{s}}, \\
& \frac{\mathrm{d} \eta_{\mathrm{s}}}{\mathrm{d} t}=v_{\mathrm{s}}, \\
& \frac{\mathrm{d} u_{\mathrm{s}}}{\mathrm{d} t}=K \theta\left(u-u_{\mathrm{s}}\right) D,
\end{aligned}
$$

$$
\begin{aligned}
\frac{\mathrm{d} v_{\mathrm{s}}}{\mathrm{d} t}= & K \theta\left(v-v_{\mathrm{s}}\right) D-\kappa\left(u-u_{\mathrm{s}}\right) \\
& \times H\left(K \theta \rho\left|\frac{\partial u}{\partial \eta}\right|\right)^{1 / 2}-\omega
\end{aligned}
$$

$$
\begin{aligned}
& \frac{\mathrm{d} T_{\mathrm{s}}}{\mathrm{d} t}=\frac{2 \chi}{3 P r} K \theta\left(T-T_{\mathrm{s}}\right) G, \\
& \frac{1}{n_{\mathrm{s}}\left(t, x_{0}\right)}=|J|, \\
& J=u_{\mathrm{s}} \frac{\partial \eta_{\mathrm{s}}\left(t, x_{0}\right)}{\partial x_{0}}-v_{\mathrm{s}} \frac{\partial x_{\mathrm{s}}\left(t, x_{0}\right)}{\partial x_{0}},
\end{aligned}
$$

where $J$ is the Jacobian of the transformation from the Eulerian to Lagrangian variables. Equations (3) contain another four dimensionless parameters related to the particles. They are defined as follows: $\kappa=(6.46 / 12 \pi \sqrt{6})\left(2 \rho_{\mathrm{s}}^{0} / \rho_{+}^{*}\right)^{1 / 4} R e_{0}^{3 / 2}$, $\omega=\left(g^{*} \sigma^{*} / 108 u_{+}^{* 2}\right)\left(2 \rho_{\mathrm{s}}^{0} / \rho_{+}^{*}\right)^{3 / 2} R e_{0}^{2}, \chi=c_{\mathrm{s}}^{*} / c_{p}^{*}$ and $R e_{0}=$ $2 u_{+}^{*} \rho_{+}^{*} \sigma^{*} / \mu_{+}^{*}$. Here $g^{*}$ is the gravity acceleration, $\rho_{\mathrm{s}}^{0}$ and $c_{\mathrm{s}}^{*}$ are the density and the special heat of the particle material, and $R e_{0}$ is the characteristic Reynolds number of the particles. It should be noted that, in addition to the interphase forces, the gravity force is included in the equation of particle motion. The boundary conditions for the particle motion take the form:

$$
\begin{array}{lll}
t=0: & x_{\mathrm{s}}=x_{0}, & \eta_{\mathrm{s}}=0, \quad u_{\mathrm{s}}=a, \\
v_{\mathrm{s}} \rightarrow 0, & T_{\mathrm{s}}=a, & n_{\mathrm{s}} v_{\mathrm{s}}=1 .
\end{array}
$$


Here particle rebounding from the wall after its deposition is ignored.

\section{Numerical method}

When the mass loading ratio of the dispersed phase $\alpha$ takes a finite value, the source terms in the right side of Eqs.(1) can not be neglected as in the one-way coupling model [11-13]. The rate of intephase exchanges depend on the velocity slip and the temperature jump between the two phases. Following the full Lagrangian approach, the particle parameters along a fixed trajectory can be obtained from Eqs.(3) with the gas flow parameters assumed to be known. In Eqs.(3), the first five are ordinary differential equations, which are solved by the fourth-order Runge-Kutta method for each specified value $x_{0}$. The last (continuity) equation is an algebraic relation from which the number density of the particles $n_{\mathrm{s}}$ can be easily obtained once the Jacobian is calculated. For doing this, four ordinary differential equations, which are obtained by differentiating the first four equations of Eqs.(3) with respect to $x_{0}$, are introduced:

$$
\begin{aligned}
\frac{\mathrm{d} w_{1}}{\mathrm{~d} t}= & w_{2}, \\
\frac{\mathrm{d} w_{3}}{\mathrm{~d} t}= & w_{4}, \\
\frac{\mathrm{d} w_{2}}{\mathrm{~d} t}= & K\left(u-u_{\mathrm{s}}\right) D \frac{\partial \theta}{\partial x_{0}}+K \theta\left(\frac{\partial u}{\partial x_{0}}-w_{2}\right) D \\
& +K \theta\left(u-u_{\mathrm{s}}\right) \frac{\partial D}{\partial x_{0}}, \\
\frac{\mathrm{d} w_{4}}{\mathrm{~d} t}= & K\left(v-v_{\mathrm{s}}\right) D \frac{\partial \theta}{\partial x_{0}}+K \theta\left(\frac{\partial v}{\partial x_{0}}-w_{4}\right) D \\
& +K \theta\left(v-v_{\mathrm{s}}\right) \frac{\partial D}{\partial x_{0}} \\
& -\kappa\left(u-u_{\mathrm{s}}\right) \frac{\partial}{\partial x_{0}}\left[\left(K \theta \rho\left|\frac{\partial u}{\partial \eta}\right|\right)^{1 / 2} H\right] \\
& +\kappa\left(K \theta \rho\left|\frac{\partial u}{\partial \eta}\right|\right)^{1 / 2} H\left(\frac{\partial u}{\partial x_{0}}-w_{2}\right) .
\end{aligned}
$$

In Eqs.(5), four new functions are defined: $w_{1}=\partial x_{\mathrm{s}} / \partial x_{0}$, $w_{2}=\partial u_{\mathrm{s}} / \partial x_{0}, w_{3}=\partial \eta_{\mathrm{s}} / \partial x_{0}$, and $w_{4}=\partial v_{\mathrm{s}} / \partial x_{0}$. The initial conditions for the above equations are as follows

$t=0: \quad w_{1}=1, \quad w_{2}=0, \quad w_{3}=0, \quad w_{4}=0$.

This full Lagrangian method offers substantial advantages over the ordinary Lagrangian methods in computation accuracy and efficiency [20]. The gas equations are numerically solved by using a finite central-difference scheme and an iteration procedure with respect to the terms describing the interphase exchange, in which the gas parameters in each Euler cell $(i, j)$, such as $u_{i j}^{n}, v_{i j}^{n}, T_{i j}^{n}, \rho_{i j}^{n}, \theta_{i j}^{n}$ (here the superscript $n$ refers to the $n$-th iteration), are repeatedly calculated. The initial flow fields $(n=0) u_{i j}^{0}, v_{i j}^{0}, T_{i j}^{0}, \rho_{i j}^{0}$ and $\theta_{i j}^{0}$ are obtained from the one-way coupling model; i.e. for the pure gas flow. Then we calculate the dispersed-phase parameters and the source terms in the carrier-phase equations and update the carrier-phase parameters. These iterations repeated until convergence.

\section{Computational results}

In the present work, numerical calculations were performed for a typical shock Mach number $(M a=2.0)$ and for aluminum powders $\left(\rho_{\mathrm{s}}^{0}=2.7 \mathrm{~g} / \mathrm{cm}^{3}\right)$ with two different diameters $\left(2 \sigma^{*}=10\right.$ or $\left.1 \mu \mathrm{m}\right)$. The other computational conditions were: $K=P r=\chi=1$ and $\gamma=1.4$. To restrict our consideration only to the laminar boundary layer region, the calculation domain was limited to $x=1$ for the $10 \mu \mathrm{m} \mathrm{par}-$ ticles and $x=100$ for the $1 \mu \mathrm{m}$ particles, respectively, on the basis of the boundary layer theory [21].

Figures 1-3 show the profiles of the gas parameters at the distance $x=0.4$ for the $10 \mu \mathrm{m}$ particles with four different values of particle mass loading $(\alpha=0,0.01,0.1,0.5)$.The results for $\alpha=0$ can be considered as those in one-way coupling case. Clearly, the presence of the particles results



Fig. 1 Horizontal velocity of the carrier phase at $x=0.4$ for $2 \sigma^{*}=$ $10 \mu \mathrm{m}$

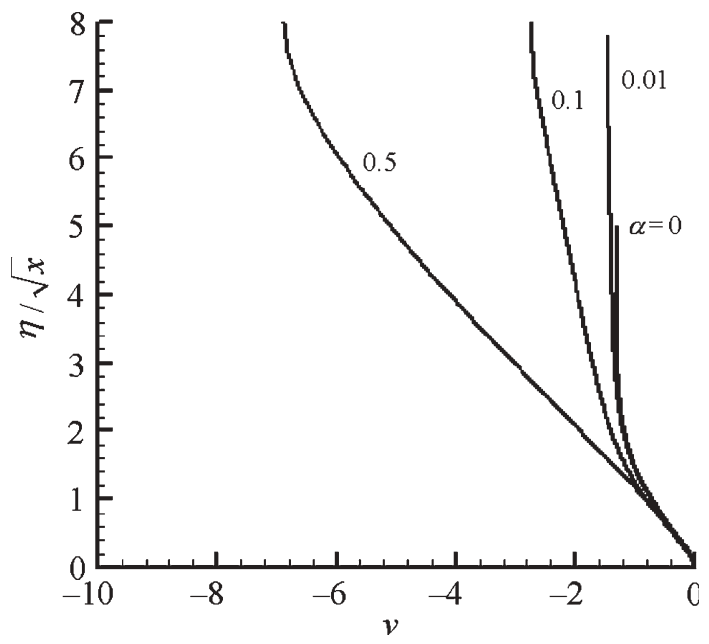

Fig. 2 Normal velocity of the carrier phase at $x=0.4$ for $2 \sigma^{*}=10 \mu \mathrm{m}$ 


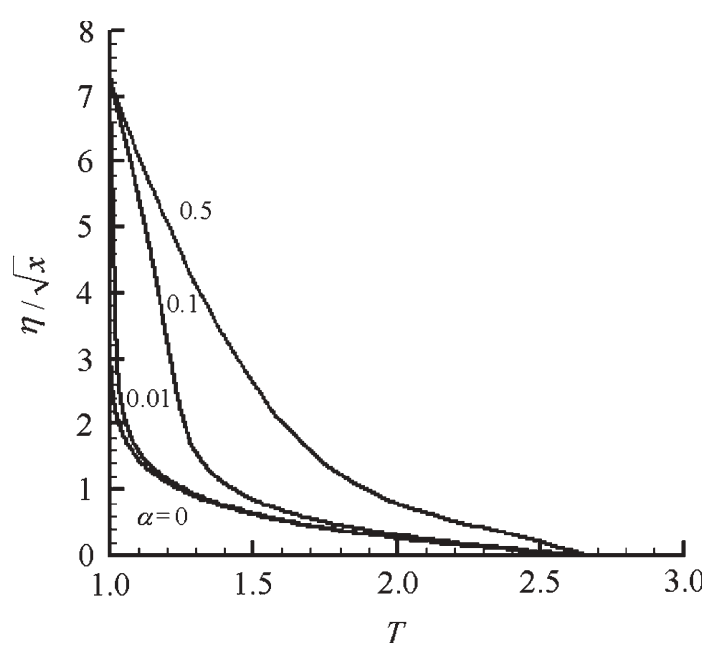

Fig. 3 Temperature of the carrier phase at $x=0.4$ for $2 \sigma^{*}=10 \mu \mathrm{m}$

in significant changes in the gas flow field. In the $10 \mu \mathrm{m}$ particles case, the effect of particles on the gas flow is appreciable at $\alpha=0.01$. Clearly, compared to the $\alpha=0$ case, the horizontal/normal velocities and the temperature as well as the boundary-layer thickness of the carrier phase increase in the two-way coupling model. With increase in $\alpha$, the coupling effect becomes more pronounced and the modification of the gas boundary layer flow due to the presence of the particles should be taken into account when the particle mass loading takes finite values. Figure 4 presents the gas horizontal velocity profiles at the distance $x=40$ for the $1 \mu \mathrm{m}$ particles with four different values of particle mass loading $(\alpha=0,0.001,0.01,0.05)$. Similarly, the coupling effect of the dispersed phase increases with the increase of the particle mass loading ratio. Besides, it is found that, for a fixed shock Mach number, small particles have a stronger impact on the gas flow. As follows from the computations, when the mass loading ratio of the $1 \mu \mathrm{m}$ particles is one-order lower than

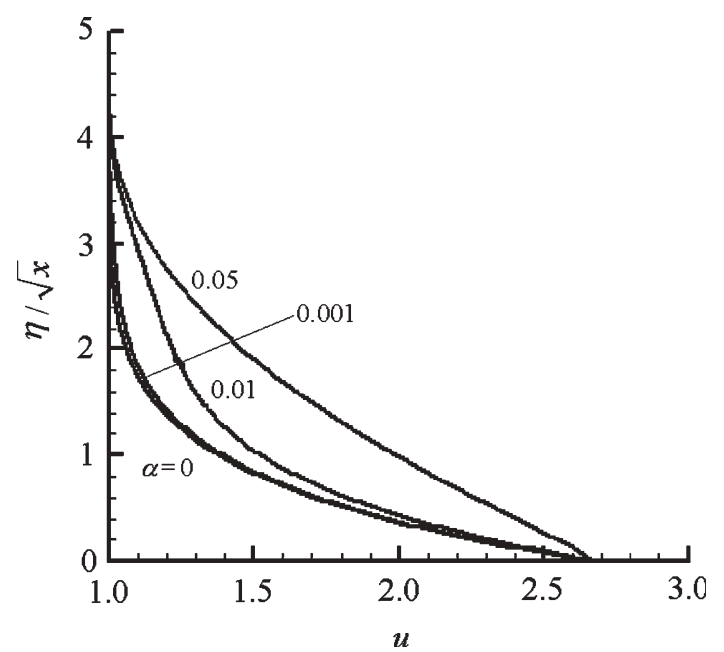

Fig. 4 Horizontal velocity of the carrier phase at $x=40$ for $2 \sigma^{*}=$ $1 \mu \mathrm{m}$

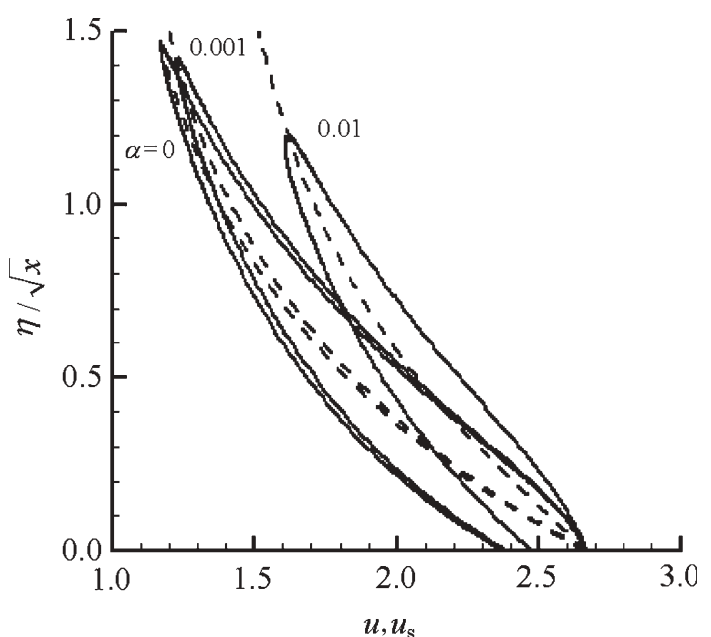

Fig. 5 Horizontal velocity of the dispersed phase at $x=80$ for $2 \sigma^{*}=$ $1 \mu \mathrm{m}$ (The dashed curves show the profiles of the carrier phase)

that of the $10 \mu \mathrm{m}$ particles, both the large and small particles affect the gas parameters rather similarly. It is attributable to the fact that, as compared to large particles, small particles have larger surface area per unit volume and hence produce a stronger effect on the carrier gas. Figures 5-7 show the flow profiles of the $1 \mu \mathrm{m}$ particles at $x=80$ for three different values $\alpha=0,0.001,0.01$. These results indicate that, at a fixed point of space, the particle parameters are two-valued, since two particle trajectories cross over inside the dust-laden gas boundary layer. In the figures, the right branches of the curves represent the particles with positive normal velocities, reflecting the action of the Saffman force. It is also clear from Figs.5-7 that, as compared to the $\alpha=0$ case, the coupling effect can yield appreciable changes of the particles flow parameters and these changes increase with the particle mass loading $\alpha$. By comparing the gas flow profiles given in Figs.1-3 with those in Figs.5-7 (see the dashed lines), it is also

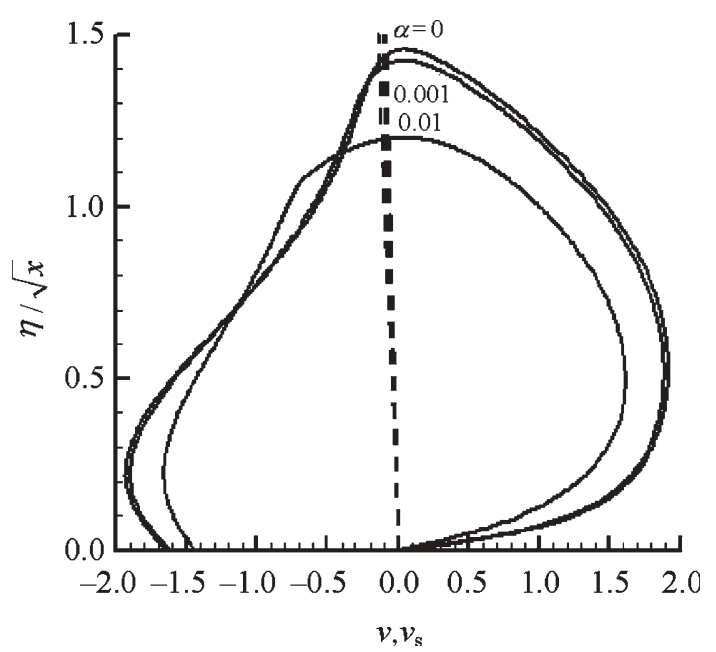

Fig. 6 Normal velocity of the dispersed phase at $x=80$ for $2 \sigma^{*}=$ $1 \mu \mathrm{m}$ (The dashed curves show the profiles of the carrier phase) 


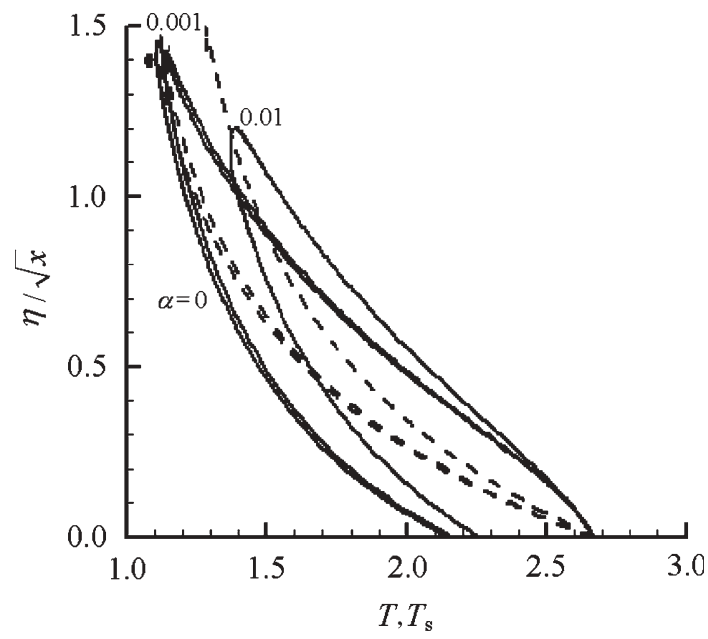

Fig. 7 Temperature of the dispersed phase at $x=80$ for $2 \sigma^{*}=1 \mu \mathrm{m}$ (The dashed curves show the profiles of the carrier phase)

found that, with the increase of the distance from the shock front downstream, the particle effect on the gas flow becomes more pronounced. In Fig.8, for the $1 \mu \mathrm{m}$ particles and three values of $\alpha=0,0.001$ and 0.01 , we have plotted the profiles of the Jacobian $J$ at the distance $x=80$. As before, the right branch corresponds to ascending particles and the left one to descending particles. From the Lagrangian continuity equation for the dispersed phase we have $|J|=1 / n_{\mathrm{s}}$ and the number density of the dispersed phase can be easily obtained. The real number density of the dispersed phase at any Euler cell is equal to the sum of the density value calculated along the two branches at the same height. It is interesting that $J=0$ (and hence $n_{\mathrm{s}}$ grows without bound) at two levels: one is on the wall and the other on the upper boundary of the suspended-particle layer. This indicates the formation of local particle accumulation zones due to a vanishing normal velocity of the particles. This effect may be important

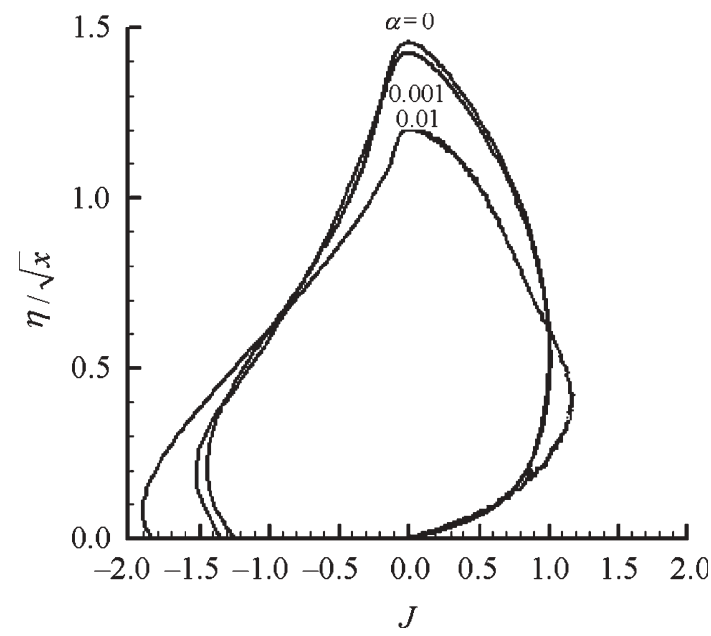

Fig. 8 Jacobian of the Eulerian-Lagrangian transformation for the dispersed phase at $x=80$ for $2 \sigma^{*}=1 \mu \mathrm{m}$ for estimating the concentration limits of explosive dust-gas mixtures.

\section{Conclusion}

A numerical two-way coupling model is developed for dusty gas laminar boundary-layer flows induced by a shock wave moving over a flat surface deposited with fine particles. The model takes into account the possibility of intersections of particle trajectories, as well as the finiteness of the particle Reynolds and Knudsen numbers. By using the finite-difference method for the gas, the full Lagrangian method for the particles, and global iterations with respect to the terms describing the interphase exchanges, the flow patterns of the carrier and dispersed phases are obtained for finite mass concentrations of the particles. The numerical results show that the Saffman force leads to the transverse migration of the deposited particles and the formation of local particle accumulation zones inside the boundary layer. When the mass loading ratio of the dispersed phase is not negligible, consideration of the two-way coupling effect results in a significant increase in the boundary-layer thickness and noticeable quantitative changes in the distribution of the flow parameters of both phases.

\section{References}

1. Zhang, F., Gronig, H., van de Ven, A.: DDT and detonation waves in dust-air mixtures. Shock Waves, 11, 53-71 (2001)

2. Bielert, U., Sichel, M.: Numerical simulation of dust explosions in pneumatic conveyors. Shock Waves 9, 125-139 (1999)

3. Gerrard, J.H.: An experimental investigation of the initial stages of the dispersion of dust by shock waves. Brit. J. Appl. Phys. 14, 186-192 (1963)

4. Borisov, A.A.: Instability of a loose material surface when the shock and detonation waves are sliding on it, Fizika Goreniya I Vzryya 3, 149-151 (1967)

5. Merzkirch, W., Bracht, K.: Erosion of dust by shock wave in air: initial stages with laminar flow. Int. J. Multiphase Flow 4, 89-95 (1978)

6. Hwang, C.C.: Initial stages of the interaction of a shock wave with a dust deposit. Int. J. Multiphase Flow 12, 655-666 (1986)

7. Boiko, V.M., Napyrin, A.N.: Dynamics of formation of a dust suspension behind a shock wave sliding over a surface of loose material. Fizika Goreniya I. Vzryva, 23, 122-126 (1987)

8. Wu, Q., Wang, B.Y.: Numerical analysis of dust particle entrainment induced by shock waves. In: Zhang G.R. (ed.), Proceedings 2nd international symposium on intense dynamic loading and its effects, 1992, pp 167-170

9. Thevand, N., Daniel, E.: Numerical study of the lift force influence on two-phase shock tube boundary layer characteristics. Shock Waves 11, 279-288 (2002)

10. Osiptsov, A.N.: Modified Lagrangian method for calculating the particle concentration in dusty-gas flows with intersecting particle trajectories. In: Proceedings 3rd International Conference on Mulyiphase Flow, ICMF'98, Lyon, France, 1998, Paper 236

11. Wang, B.Y., Osiptsov, A.N.: Near-wall boundary layer behind a shock wave in a dusty gas. Izv. Ross. Akad. Nauk, Mekh. Zhidk. Gaza 4, 61-73 (1999); Engl. Transl.: Fluid Dynamics 4, 505-515 (1999)

12. Wang, B.Y., Xiong, Y., Osiptsov, A.N.: Shock-induced dust cloud over a deposit layer of fine particles. In: Jiang Z. (ed.), Proceedings 24th International Symposium on Shock Waves, 2004, pp 10911096 
13. Wang, B.Y., Xiong, Y., Chen, Q., Osiptsov, A.N.: Dust-cloud structures behind a shock wave moving over a deposited layer of fine particles. Prog. Nat. Sci. 15, 32-41 (2005)

14. Marble, F.E.: Dynamics of dusty gases. Annu. Rev. Fluid Mech. 2 , 397-446 (1970)

15. Carlson, D.J., Hoglund, R.F.: Particle drag and heat transfer in rocket nozzles. AIAA J. 2, 1980-1984 (1964)

16. Fush, N.A.: Mechanics of aerosols. New York: MacMillan, 1964

17. Saffman, P.G.: The lift on a small sphere in a slow shear flow. J. Fluid Mech. 22, 384-400 (1965); and Corrigendum. J. Fluid Mech. 31, 624 (196-8)
18. Mei, R.: An approximate expression for the shear lift force on a spherical particle at finite Reynolds number. Int. J. Multiphase Flow 18, 145-147 (1992)

19. Rudinger, G.: Fundamentals of gas-particle flow. Amsterdam: Elservier, 1980

20. Healy, D.P., Young, J.B.: Calculation of inertial particle transport using the Osiptsov Lagrangian method. In: ICMF2001, New Orleans, 2001, CD: 1-12

21. Schlichting H.: Boundary Layer Theory, 7th edition. New York: McGraw-Hill, 1979 